\title{
Duration of breast-feeding and the incidence of type 2 diabetes mellitus in the Shanghai Women's Health Study
}

\author{
R. Villegas • Y.-T. Gao • G. Yang • H. L. Li • T. Elasy • \\ W. Zheng $\cdot$ X.-O. Shu
}

Received: 18 July 2007 / Accepted: 22 October 2007 / Published online: 27 November 2007

(C) Springer-Verlag 2007

\begin{abstract}
Aims/hypothesis The aim of this study was to examine the association between lifetime breast-feeding and the incidence of type 2 diabetes mellitus in a large populationbased cohort study of middle-aged women.

Methods This was a prospective study of 62,095 middleaged parous women in Shanghai, China, who had no prior history of type 2 diabetes mellitus, cancer or cardiovascular disease at study recruitment. Breast-feeding history, dietary intake, physical activity and anthropometric measurements were assessed by in-person interviews. The Cox regression model was employed to evaluate the association between breast-feeding and the risk of type 2 diabetes mellitus.

Results After 4.6 years of follow-up, 1,561 women were diagnosed with type 2 diabetes mellitus. Women who had breastfed their children tended to have a lower risk of diabetes mellitus than those who had never breastfed [relative risk $(\mathrm{RR})=0.88 ; 95 \% \mathrm{CI}, 0.76-1.02 ; p=0.08]$. Increasing duration of breast-feeding was associated with a reduced risk of type 2 diabetes mellitus. The fully adjusted RRs for lifetime breastfeeding duration were $1.00,0.88,0.89,0.88,0.75$ and 0.68
\end{abstract}

R. Villegas $\cdot$ G. Yang $\cdot$ W. Zheng $\cdot$ X.-O. Shu $(\bowtie)$

Vanderbilt Epidemiology Center,

Institute for Medicine and Public Health,

Department of Medicine, Vanderbilt University Medical Center,

2525 West End Avenue, Suite 600,

Nashville, TN 37232-1738, USA

e-mail: xiao-ou.shu@vanderbilt.edu

Y.-T. Gao $\cdot$ H. L. Li

Department of Epidemiology, Shanghai Cancer Institute,

Shanghai, People's Republic of China

T. Elasy

Diabetes Research and Training Center,

Vanderbilt University Medical Center,

Nashville, TN, USA
( $p$ trend $=0.01$ ) for $0,>0$ to $0.99,>0.99$ to $1.99,>1.99$ to 2.99 , $>2.99$ to 3.99 and $\geq 4$ years in analyses adjusted for age, daily energy intake, BMI, WHR, smoking, alcohol intake, physical activity, occupation, income level, education level, number of live births and presence of hypertension at baseline.

Conclusions/interpretation Breast-feeding may protect parous women from developing type 2 diabetes mellitus later in life.

Keywords Lifelong breast-feeding · Middle-aged women . Parous women · Type 2 diabetes mellitus
Abbreviations
MET metabolic equivalents
RR relative risk
SWHS Shanghai Women's Health Study

\section{Introduction}

The prevalence of type 2 diabetes mellitus has been increasing rapidly worldwide [1], making knowledge of risk factors and protective factors associated with type 2 diabetes mellitus essential for the development of prevention strategies.

Results from animal and human studies suggest an improvement in glucose and insulin sensitivity during lactation [2-5]. Data from two large cohorts in the USA also indicate that longer duration of breast-feeding may reduce the risk of type 2 diabetes mellitus by improving glucose homeostasis [6].

We examined the association between lifetime breastfeeding and the incidence of type 2 diabetes mellitus in a large population-based cohort study of middle-aged women, the Shanghai Women's Health Study (SWHS). 


\section{Methods}

Study population The SWHS is a population-based prospective cohort study of middle-aged women (40-70 years old) conducted in seven urban communities of Shanghai, China. Details of the SWHS survey have been reported elsewhere [7]. From a total of 81,170 women who were invited to participate, 75,221 were recruited $(92.7 \%$ participation rate). Reasons for non-participation were refusal (3.0\%), absence during the enrolment period $(2.6 \%)$ and other reasons (health, hearing, speaking problems; $1.6 \%$ ). After exclusion of women younger than 40 years or older than 70 years at the time of interview $(n=278), 74,942$ women remained for the study. Participants completed a detailed survey as part of an in-person interview, which included assessment of dietary intake, physical activity, measurement of anthropometrics and other lifestyle factors. The original questionnaires, in Chinese, are available from the corresponding author upon request. Protocols for the SWHS were approved by the Institutional Review Boards of all institutes involved in the study and written informed consent was obtained prior to interview. Biannual in-person follow-up of all living cohort members was conducted by in-home visit from 2000 to 2002 and from 2002 to 2004, with a response rate of 99.8 and $98.7 \%$, respectively; only 934 participants were lost to follow-up.

Outcome ascertainment Incident type 2 diabetes mellitus was identified through follow-up surveys. A total of 1,561 parous women reported a type 2 diabetes mellitus diagnosis since the baseline survey. For the current study we considered a case of type 2 diabetes mellitus to be confirmed if the participants reported having been diagnosed with type 2 diabetes mellitus and met at least one of the following criteria: fasting glucose level $\geq 7 \mathrm{mmol} / \mathrm{l}$ on at least two separate occasions or an oral glucose tolerance test with a value $\geq 11.1 \mathrm{mmol} / 1$ and/or use of hypoglycaemic medication (i.e. insulin or oral hypoglycaemic drugs). All tests were performed as part of patients' primary care. Of the self-reported cases, a total of 869 participants met the study outcome criteria and are referred to herein as confirmed cases of type 2 diabetes mellitus. We performed analyses restricted to confirmed cases as well as analyses including all cases of type 2 diabetes mellitus.

Breast-feeding duration assessment Information on each pregnancy was obtained during the in-person interview. This included date and outcome of each pregnancy and whether and for how long the participant breastfed each child. Based on this information, we calculated the duration of breast-feeding (months and years) and duration of breastfeeding per live birth (years).
Measurement of potential confounders Anthropometric measurements were taken at baseline recruitment according to a standard protocol by trained interviewers who were retired medical professionals [8]. Self-reported body weight history was obtained for ages 20 and 40 years and at baseline recruitment. We calculated BMI (weight in $\mathrm{kg}$ divided by the square of height in $\mathrm{m}$ ), WHR (waist circumference divided by hip circumference) and standardised weight change (difference between measured weight at baseline and weight at 20 years divided by the interval between study recruitment and 20 years; $\mathrm{kg} /$ year).

A structured questionnaire was used at the baseline survey to collect information on socio-demographic factors such as age, level of education (none, elementary school, middle/high school, college), family income in yuan per year $(<10,000,10,000-19,999,20,000-29,999,>30,000)$, occupation (professional, clerical, manual labour/other, housewife/retired), smoking (smoked at least one cigarette per day for more than 6 months continuously) and alcohol consumption (had ever drunk beer, wine or spirits at least three times per week). History of diseases such as diabetes, cancer, cardiovascular disease and high blood pressure was also collected.

Information on physical activity was obtained using a validated questionnaire [9]. The questionnaire evaluated exercise and sport participation, daily activity and daily commuting round-trip journey to work. We calculated the metabolic equivalents (METs) for each activity, using a compendium of physical activity values [10]. We derived a quantitative estimate of overall non-occupational activity (MET-h per day).

Dietary intake was assessed through an in-person interview using a validated food frequency questionnaire at the baseline recruitment survey and at the first follow-up survey [11]. The Chinese food composition tables [12] were used to estimate energy intake $(\mathrm{kJ} /$ day).

Statistical analysis Person-years for each participant were calculated as the interval between the baseline recruitment to one of the following: diagnosis of type 2 diabetes mellitus; censored at death; or completion of the second follow-up survey.

The Cox proportional hazards model was used to assess the effect of breast-feeding on the incidence of type 2 diabetes mellitus. Tests for trend were performed by entering the categorical variables as continuous parameters in the models. In all models, we adjusted for the following potential confounding variables: age, BMI, WHR, total energy, physical activity, number of live births (entered as continuous variables), income level, education level, occupation, smoking status, alcohol consumption status and presence of hypertension at baseline (as categorical variables). 
We also derived a propensity score by regressing breastfeeding (yes/no) on risk factors for type 2 diabetes mellitus (age, daily energy intake, BMI, WHR, smoking, alcohol consumption, physical activity, hypertension, income, education level, occupation status, oral contraceptive use, vegetable intake, legume intake, meat intake and staples intake). We then examined the association between breastfeeding (yes/no) and type 2 diabetes mellitus by including the propensity score in the Cox proportional hazard model. All analyses were performed using SAS (version 9.1, SAS Institute, Cary, NC, USA), and all tests of statistical significance were based on two-sided probability. A $p$ value of less than 0.05 was considered statistically significant.

\section{Results}

The average age of the entire SWHS cohort $(n=74,942)$ was $52.1(\mathrm{SD}=9.1)$. The average age at first pregnancy was 25.5 years (median: 25.7 years) and the median number of

Table 1 Age-standardised characteristics of parous women in the Shanghai Women's Health Study by duration of breast-feeding

\begin{tabular}{|c|c|c|c|c|c|c|}
\hline & \multicolumn{5}{|c|}{ Duration of breast-feeding (months) } & \multirow[b]{2}{*}{$\begin{array}{l}p \text { value for } \\
\text { trend }^{\text {a }}\end{array}$} \\
\hline & 0 & $>0$ to 6 & $>6$ to 11 & $>11$ to 35 & $\geq 36$ & \\
\hline Women $(n)$ & 11,395 & 10,463 & 14,958 & 18,144 & 7,135 & \\
\hline Cases of diabetes $(n)$ & 225 & 163 & 253 & 585 & 335 & \\
\hline Person-years $(n)$ & 53,038 & 48,770 & 69,741 & 83,870 & 32,535 & \\
\hline$<10$ years since last pregnancy $(\%)$ & 26.9 & 21.9 & 28.9 & 18.6 & 3.7 & \\
\hline Age, median (Q25-Q75) & $45(42-49)$ & $45(42-49)$ & $45(42-49)$ & $54(49-61)$ & $64(62-67)$ & $<0.001$ \\
\hline Energy intake, mean kJ/day (SE) & $6,875(14)$ & $6,927(15)$ & $6,920(13)$ & $6,985(11)$ & $6,738(21)$ & $<0.001$ \\
\hline Live births, median (Q25-Q75) & $1(1-1)$ & $1(1-1)$ & $1(1-1)$ & $2(1-2)$ & $4(3-5)$ & $<0.001$ \\
\hline BMI, mean $\mathrm{kg} / \mathrm{m}^{2}(\mathrm{SE})$ & $23.4(0.03)$ & $23.2(0.03)$ & $23.6(0.03)$ & $24.2(0.02)$ & $24.7(0.04)$ & $<0.001$ \\
\hline WHR, mean (SE) & $0.80(0.0005)$ & $0.8(0.0005)$ & $0.80(0.0005)$ & $0.81(0.004)$ & $0.82(0.007)$ & $<0.001$ \\
\hline Weight gain, mean kg/year (SE) & $0.34(0.003)$ & $0.31(0.003)$ & $0.33(0.003)$ & $0.36(0.003)$ & $0.37(0.005)$ & $<0.001$ \\
\hline $\begin{array}{l}\text { Weight at age } 20 \text { years, median } \mathrm{kg} \\
\text { (Q25-Q75) }\end{array}$ & $48(45-53)$ & $49(45-53)$ & $50(45-54)$ & $50(45-54)$ & $50(45-55)$ & $<0.001$ \\
\hline Smoker $(\%)$ & $1.7(1.5-1.9)$ & $1.4(1.2-1.9)$ & $1.5(1.3-1.7)$ & $2.4(2.3-2.5)$ & $3.6(3.2-4.0)$ & $<0.001$ \\
\hline Ever drinker (\%) & $1.9(1.7-2.1)$ & $2.1(1.9-2.3)$ & $2.1(1.9-2.3)$ & $2.4(2.3-2.5)$ & $1.9(1.7-2.1)$ & $<0.001$ \\
\hline Exercise $(\%)$ & $30.5(29.5-31.5)$ & $32.9(31.9-33.9)$ & $34.3(33.3-35.3)$ & $33.7(32.7-34.7)$ & $31.4(30.4-32.4)$ & 0.18 \\
\hline High physical activity ${ }^{\mathrm{b}}(\%)$ & $22.8(22.0-23.6)$ & $22.1(21.3-22.9)$ & $24.4(21.6-25.2)$ & $27.3(26.9-27.7)$ & $41.2(40.2-42.2)$ & $<0.001$ \\
\hline \multicolumn{7}{|l|}{ Education $(\%)$} \\
\hline None & $11.0(12.4-11.6)$ & $8.5(7.9-9.1)$ & $9.6(9.0-10.2)$ & $17.8(17.4-18.2)$ & $41.2(40.2-42.2)$ & $<0.001$ \\
\hline Elementary & $38.2(37.2-39.2)$ & $34.4(33.4-35.4)$ & $39.2(38.2-40.2)$ & $44.3(43.8-44.8)$ & $46.9(47.9-48.9)$ & \\
\hline Middle/high school & $32.5(31.5-33.5)$ & $34.3(33.3-35.3)$ & $32.4(31.4-33.4)$ & $27.1(26.7-27.5)$ & $8.6(8.0-9.2)$ & \\
\hline College & $18.3(17.5-19.1)$ & $22.7(21.9-23.5)$ & $18.8(18.0-19.6)$ & $10.8(10.5-11.1)$ & $3.3(2.9-3.7)$ & \\
\hline \multicolumn{7}{|l|}{ Household income (\%) } \\
\hline$<10,000$ yuan & $14.0(13.4-14.6)$ & $11.6(11.0-12.2)$ & $12.6(12.0-13.2)$ & $15.6(15.3-15.9)$ & $22.1(21.3-22.9)$ & $<0.001$ \\
\hline $10,000-19,999$ yuan & $38.8(37.8-39.8)$ & $35.8(34.8-36.8)$ & $37.1(36.1-38.1)$ & $38.0(37.5-38.5)$ & $38.7(37.7-39.7)$ & \\
\hline $20,000-29,999$ yuan & $29.4(28.5-30.3)$ & $31.3(30.3-32.3)$ & $30.4(29.4-31.4)$ & $28.6(28.2-29.0)$ & $27.8(27.0-28.6)$ & \\
\hline$>30,000$ yuan & $17.8(17.0-18.6)$ & $21.4(20.6-22.4)$ & $19.8(19.0-20.6)$ & $17.8(17.4-18.2)$ & $11.4(10.8-12.0)$ & \\
\hline \multicolumn{7}{|l|}{ Occupation (\%) } \\
\hline Professional & $21.6(20.8-22.4)$ & $25.9(25.1-26.7)$ & $22.2(21.4-23.0)$ & $16.4(15.6-17.2)$ & $6.8(6.2-7.4)$ & $<0.001$ \\
\hline Clerical & $12.8(12.2-13.4)$ & $12.4(11.6-12.8)$ & $13.2(12.6-13.8)$ & $13.7(13.4-14.0)$ & $19.5(18.7-20.3)$ & \\
\hline Manual labour/others & $21.8(21.0-22.6)$ & $21.0(20.2-21.8)$ & $23.1(22.3-23.9)$ & $23.6(23.2-24.0)$ & $20.0(19.2-20.8)$ & \\
\hline Housewife/retired & $43.8(42.8-44.8)$ & $40.7(39.7-41.7)$ & $41.6(40.6-42.6)$ & $46.3(45.8-46.8)$ & $53.6(52.6-54.6)$ & \\
\hline Hypertension (\%) & $18.4(17.6-19.2)$ & $18.3(17.5-19.1)$ & $18.8(18.0-19.6)$ & $19.8(19.4-20.2)$ & $16.6(15.8-17.4)$ & 0.12 \\
\hline
\end{tabular}

Data are presented as $n$, percent, median (Q25-Q75, interquartile range) and mean (SE), and are directly standardised in years to the age distribution of the population

Means of energy intake, BMI, WHR and standard weight are adjusted by age

Weight at 20 years and number of live births were not normally distributed and could not be adjusted for age

${ }^{a} p$ values for trend were calculated by: proportional odds model for prevalence of population characteristics; ANOVA test for daily energy intake, BMI, WHR and standard weight gain; and Kruskal-Wallis test for age, number of live births and weight at 20 years

${ }^{\mathrm{b}}$ High physical activity: participants in the upper quartile of total METs 
live births was 1.0. A total of $3.3 \%$ women had no children, $54.4 \%$ had only one child, $21.2 \%$ had two children, $10.5 \%$ had three children, $6.2 \%$ had four children and $4.3 \%$ had five or more children. The incidence of diabetes mellitus was 6.0 cases per 1,000 person-years at risk.

There were 62,095 parous women in this study with no prior history of type 2 diabetes mellitus, cancer or cardiovascular disease at study recruitment; of these 50,700 $(81.65 \%)$ reported having breastfed their children. The average number of live births was 1.7 , the average number of months of breast-feeding was 14.6 and the average number of years of breast-feeding per child was 0.6 . The number of live births was associated with months of breastfeeding (Spearman correlation coefficient, $0.70 ; p<0.001$ ). Age-standardised characteristics of the study population by duration of breast-feeding are shown in Table 1. Women with a longer duration of breast-feeding were older, had less education, lower income and were less likely to be employed at the time of the survey or to hold a professional job. Duration of breast-feeding was also associated with smoking and higher physical activity in this population. We did not find an association between differences in participation in exercise or prevalence of hypertension at baseline and duration of breast-feeding $(p>0.05)$.

Women who had breastfed tended to have a lower risk of type 2 diabetes mellitus [relative risk $(\mathrm{RR})=0.88 ; 95 \% \mathrm{CI}$, $0.76-1.02 ; p=0.08]$ than women who had never breastfed, in analyses adjusted for age, daily energy intake, BMI, WHR, number of live births, occupation, income levels, education, smoking, alcohol consumption, physical activity and presence of hypertension (Table 2). When we adjusted the analysis by a propensity score of predictors of type 2 diabetes mellitus, the RR was $0.81(95 \%$ CI, 0.70-0.94; $p<0.01$ ).

The duration of breast-feeding and duration of breastfeeding per child were associated with a lower risk of type 2 diabetes mellitus (Table 2). The fully adjusted RR for 0 , $>0$ to $0.99,>0.99$ to $1.99,>1.99$ to $2.99,>2.99$ to 3.99 and $\geq 4$ years of breast-feeding with the risk of type 2 diabetes mellitus were $1.00,0.88,0.89,0.88,0.75$ and 0.68 . The fully adjusted RR between years of breast-feeding per child and type 2 diabetes mellitus risk were 1.00, 0.91, 0.87 and 0.87 ( $p=0.11$ for trend) for $0,>0$ to $0.49,>0.49$ to 0.99 and $\geq 1$ years of breast-feeding per number of births. Associations between months of breast-feeding and incidence of type 2 diabetes mellitus are presented in Table 3. BMI and WHR are the two major confounding factors for the association between the duration of breast-feeding and risk of type 2 diabetes mellitus. Both BMI and WHR were weakly correlated with breast-feeding $(r=0.22$ and 0.22 , respectively). The correlation between BMI and WHR was 0.46 . The association between the duration of breastfeeding and diabetes mellitus was accentuated and the trend was only marginally significant when we adjusted the model for BMI. Additional adjustment for WHR resulted in the trend becoming statistically significant $(p=0.04)$. The association between breast-feeding and type 2 diabetes mellitus did not change much after adjustment for number of live births. When we restricted the analysis of breastfeeding and incidence of type 2 diabetes to confirmed cases of type 2 diabetes mellitus, we found similar patterns of association (Table 4).

Table 2 Associations between type 2 diabetes mellitus and duration of breast-feeding for all participants, Shanghai Women's Health Study

\begin{tabular}{|c|c|c|c|c|c|}
\hline & $\%$ & Cases per person-years & $\mathrm{RR}$ & $95 \% \mathrm{CI}$ & $p$ value for trend \\
\hline \multicolumn{6}{|l|}{ Breast-feeding } \\
\hline No & 18.35 & $225 / 53,038$ & 1.00 & & \\
\hline Yes & 81.65 & $1,336 / 234,916$ & 0.88 & $0.76-1.02$ & \\
\hline \multicolumn{6}{|c|}{ Duration of breast-feeding } \\
\hline \multicolumn{6}{|l|}{ Years } \\
\hline 0 & 18.35 & $225 / 53,038$ & 1.00 & & 0.01 \\
\hline$>0-0.99$ & 47.99 & $416 / 118,511$ & 0.88 & $0.75-1.04$ & \\
\hline$>0.99-1.99$ & 17.31 & $343 / 57,418$ & 0.89 & $0.75-1.06$ & \\
\hline$>1.99-2.99$ & 7.57 & $242 / 26,451$ & 0.88 & $0.71-1.07$ & \\
\hline$>2.99-3.99$ & 4.15 & $148 / 14,929$ & 0.75 & $0.59-0.96$ & \\
\hline$\geq 4$ & 4.63 & $187 / 17,606$ & 0.68 & $0.52-0.90$ & \\
\hline \multicolumn{6}{|c|}{ Years per child } \\
\hline 0 & 18.35 & $225 / 53,038$ & 1.00 & & 0.11 \\
\hline$>0-0.49$ & 15.10 & $200 / 43,615$ & 0.91 & $0.75-1.10$ & \\
\hline$>0.49-0.99$ & 44.20 & $659 / 127,453$ & 0.87 & $0.74-1.02$ & \\
\hline$\geq 1$ & 22.35 & $477 / 63,848$ & 0.87 & $0.78-1.03$ & \\
\hline
\end{tabular}

Values are adjusted for age, daily energy intake, BMI, WHR, number of live births, smoking, alcohol consumption, physical activity, education, income, occupation and hypertension 
Table 3 Incidence of type 2 diabetes by duration of breast-feeding, Shanghai Women's Health Study

\begin{tabular}{|c|c|c|c|c|c|c|c|c|c|c|c|}
\hline & \multicolumn{2}{|l|}{0} & \multicolumn{2}{|c|}{$>0$ to 6 months } & \multicolumn{2}{|c|}{$>6$ to 11 months } & \multicolumn{2}{|c|}{$>11$ to 35 months } & \multicolumn{2}{|c|}{$\geq 36$ months } & \multirow[b]{2}{*}{$p$ value for trend } \\
\hline & RR & $95 \% \mathrm{CI}$ & RR & $95 \%$ CI & RR & $95 \% \mathrm{CI}$ & RR & $95 \% \mathrm{CI}$ & $\mathrm{RR}$ & $95 \%$ CI & \\
\hline Model 1 & 1.00 & & 0.81 & $0.66-0.99$ & 0.88 & $0.74-1.07$ & 1.08 & $0.92-1.27$ & 1.03 & $0.84-1.25$ & 0.20 \\
\hline Model 2 & 1.00 & & 0.84 & $0.69-1.03$ & 0.89 & $0.74-1.07$ & 0.99 & $0.85-1.18$ & 0.88 & $0.71-1.08$ & 0.75 \\
\hline Model 3 & 1.00 & & 0.84 & $0.69-1.03$ & 0.88 & $0.73-1.05$ & 0.90 & $0.76-1.05$ & 0.76 & $0.62-0.93$ & 0.06 \\
\hline Model 4 & 1.00 & & 0.87 & $0.71-1.06$ & 0.91 & $0.76-1.09$ & 0.98 & $0.83-1.15$ & 0.84 & $0.68-1.03$ & 0.39 \\
\hline Model 5 & 1.00 & & 0.89 & $0.71-1.06$ & 0.89 & $0.74-1.07$ & 0.89 & $0.76-1.05$ & 0.75 & $0.61-0.92$ & 0.04 \\
\hline Model 6 & 1.00 & & 0.87 & $0.71-1.06$ & 0.89 & $0.75-1.07$ & 0.89 & $0.75-1.05$ & 0.73 & $0.58-0.91$ & 0.05 \\
\hline
\end{tabular}

Model 1 age-adjusted only; Model 2: age plus other confounders, but not BMI, WHR or number of live births; Model 3: model 2 plus BMI; Model 4: model 2 plus WHR; Model 5: model 2 plus BMI and WHR; Model 6: model 5 plus number of live births

In analyses restricted to women who reported having been pregnant within the last 10 years (Table 5), the RR for type 2 diabetes mellitus by the duration of breast-feeding per number of live births was $1.00,1.01,0.74$ and $0.65 ;(p=$ 0.03 for trend) for $0,>0$ to $0.49,>0.49$ to 0.99 and $\geq 1$ years of breast-feeding per number of live births. Among women who had been pregnant within the last 10 years, the RRs of type 2 diabetes mellitus for $0,>0$ to $0.99,>0.99$ to 1.99 , $>1.99$ to $2.99,>2.99$ to 3.99 and $\geq 4$ years of breast-feeding were $1.00,0.80,0.78,0.58,0.47$ and $0.45(p=0.04$ for trend). The RRs of type 2 diabetes mellitus for $0,>0$ to 0.99 , $>0.99$ to $1.99,>1.99$ to $2.99,>2.99$ to 3.99 and $\geq 4$ years of breast-feeding among women who had been pregnant within the last 5 years were $1.00,0.87,0.68,0.44,0.69$ and $\geq 0.49$ ( $p=0.02$ for trend; data not shown in tables). In addition, we found that the RRs for diabetes mellitus associated with $\geq 12$ months of breast-feeding were 0.66 (95\% CI, 0.45 0.95), 0.63 (95\% CI, 0.40-0.98), 0.49 (95\% CI, 0.28-0.85) and 0.46 (95\% CI, 0.26-0.86), respectively, when analyses were conducted with time since last pregnancy defined as $\leq 13, \leq 10, \leq 5$ and $\leq 2$ years (data not shown). In analyses restricted to women who had not been pregnant in the last 10 years, we still found inverse associations between breastfeeding and risk of type 2 diabetes mellitus, although the associations between months of breast-feeding and years of breast-feeding per child were not significant. The association between years of breast-feeding and type 2 diabetes mellitus was of marginal significance. The RRs of type 2 diabetes mellitus for $0,>0$ to $0.99,>0.99$ to $1.99,>1.99$ to 2.99 , $>2.99$ to 3.99 and $\geq 4$ years of breast-feeding were $1.00,0.90$, 0.94, 0.94, 0.79 and 0.72 ( $p=0.06$ for trend). Similar trends were found when the analysis was restricted to confirmed
Table 4 Associations between type 2 diabetes mellitus and duration of breast-feeding, Shanghai Women's Health Study (confirmed cases of diabetes only)
Values are adjusted for age, daily energy intake, BMI, WHR, number of live births, smoking, alcohol consumption, physical activity, education, income, occupation and hypertension

\begin{tabular}{|c|c|c|c|c|c|}
\hline & $\%$ & Cases per person-years & $\mathrm{RR}$ & $95 \% \mathrm{CI}$ & $p$ value \\
\hline \multicolumn{6}{|l|}{ Breast-feeding } \\
\hline No & 18.40 & $128 / 52,848$ & 1.00 & & \multirow[t]{2}{*}{0.17} \\
\hline Yes & 81.60 & $741 / 233,836$ & 0.87 & $0.72-1.06$ & \\
\hline \multicolumn{6}{|c|}{ Duration of breast-feeding } \\
\hline \multicolumn{6}{|c|}{ Months } \\
\hline 0 & 18.40 & $128 / 52,848$ & 1.00 & & \multirow[t]{5}{*}{0.17} \\
\hline$>0-6$ & 16.91 & $86 / 48,617$ & 0.80 & $0.61-1.05$ & \\
\hline$>6-11$ & 24.21 & $159 / 69,558$ & 0.98 & $0.77-1.24$ & \\
\hline$>11-35$ & 29.11 & $314 / 83,398$ & 0.84 & $0.68-1.05$ & \\
\hline$>36$ & 11.37 & $182 / 32,263$ & 0.74 & $0.55-1.01$ & \\
\hline \multicolumn{6}{|l|}{ Years } \\
\hline 0 & 18.40 & $128 / 52,848$ & 1.00 & & \multirow[t]{6}{*}{0.01} \\
\hline$>0-0.99$ & 48.20 & $297 / 11,817$ & 0.91 & $0.73-1.13$ & \\
\hline$>0.99-1.99$ & 17.25 & $200 / 57,156$ & 0.90 & $0.72-1.13$ & \\
\hline$>1.99-2.99$ & 7.48 & $99 / 26,242$ & 0.72 & $0.54-0.95$ & \\
\hline$>2.99-3.99$ & 4.10 & $65 / 14,809$ & 0.72 & $0.52-1.01$ & \\
\hline$>4$ & 4.57 & $78 / 17,454$ & 0.67 & $0.46-0.96$ & \\
\hline \multicolumn{6}{|c|}{ Years per child } \\
\hline 0 & 18.40 & $128 / 52,848$ & 1.00 & & \multirow[t]{4}{*}{0.23} \\
\hline$>0-0.49$ & 15.13 & $116 / 43,443$ & 0.94 & $0.73-1.21$ & \\
\hline$>0.49-0.99$ & 44.21 & $359 / 126,916$ & 0.84 & $0.69-1.04$ & \\
\hline$>1$ & 22.25 & $266 / 63,447$ & 0.88 & $0.70-1.11$ & \\
\hline
\end{tabular}


Table 5 Duration of breast-feeding and risk of type 2 diabetes mellitus, Shanghai Women's Health Study

\begin{tabular}{|c|c|c|c|c|c|c|}
\hline & \multicolumn{6}{|c|}{ Time since last pregnancy } \\
\hline & \multicolumn{3}{|c|}{$\leq 10$ years since last pregnancy } & \multicolumn{3}{|c|}{$>10$ years since last pregnancy } \\
\hline & $\mathrm{RR}$ & $95 \% \mathrm{CI}$ & $p$ value for trend & $\mathrm{RR}$ & $95 \% \mathrm{CI}$ & $p$ value for trend \\
\hline \multicolumn{7}{|l|}{ All participants } \\
\hline \multicolumn{7}{|l|}{ Months } \\
\hline 0 & 1.00 & & 0.05 & 1.00 & & 0.20 \\
\hline$>0-6$ & 0.90 & $0.57-1.42$ & & 0.88 & $0.70-1.10$ & \\
\hline$>6-11$ & 0.83 & $0.55-1.25$ & & 0.91 & $0.74-1.12$ & \\
\hline$>11-35$ & 0.63 & $0.40-0.98$ & & 0.94 & $0.79-1.13$ & \\
\hline$\geq 36$ & 0.66 & $0.30-1.48$ & & 0.76 & $0.60-0.97$ & \\
\hline \multicolumn{7}{|l|}{ Years } \\
\hline 0 & 1.00 & & 0.04 & 1.00 & & 0.06 \\
\hline$>0-0.99$ & 0.87 & $0.60-1.24$ & & 0.90 & $0.75-1.08$ & \\
\hline$>0.99-1.99$ & 0.68 & $0.43-1.09$ & & 0.94 & $0.77-1.13$ & \\
\hline$>1.99-2.99$ & 0.44 & $0.21-0.92$ & & 0.94 & $0.76-1.17$ & \\
\hline$>2.99-3.99$ & 0.69 & $0.29-1.62$ & & 0.79 & $0.61-1.02$ & \\
\hline$\geq 4$ & 0.49 & $0.18-1.35$ & & 0.72 & $0.54-0.96$ & \\
\hline \multicolumn{7}{|l|}{ Years per child } \\
\hline 0 & 1.00 & & 0.03 & 1.00 & & 0.45 \\
\hline$>0-0.49$ & 1.00 & $0.64-1.59$ & & 0.91 & $0.74-1.13$ & \\
\hline$>0.49-0.99$ & 0.74 & $0.51-1.07$ & & 0.90 & $0.76-1.07$ & \\
\hline$\geq 1$ & 0.65 & $0.41-1.05$ & & 0.92 & $0.76-1.10$ & \\
\hline \multicolumn{7}{|c|}{ Confirmed diabetes only } \\
\hline \multicolumn{7}{|c|}{ Months } \\
\hline 0 & 1.00 & & 0.26 & 1.00 & & 0.34 \\
\hline$>0-6$ & 0.80 & $0.41-1.53$ & & 0.81 & $0.60-1.09$ & \\
\hline$>6-11$ & 0.96 & $0.55-1.65$ & & 0.98 & $0.75-1.27$ & \\
\hline$>11-35$ & 0.69 & $0.39-1.22$ & & 0.87 & $0.69-1.11$ & \\
\hline$>36$ & 0.60 & $0.23-1.57$ & & 0.78 & $0.56-1.08$ & \\
\hline \multicolumn{7}{|l|}{ Years } \\
\hline 0 & 1.00 & & 0.10 & 1.00 & & 0.06 \\
\hline$>0-0.99$ & 0.90 & $0.55-1.48$ & & 0.91 & $0.72-1.16$ & \\
\hline$>0.99-1.99$ & 0.80 & $0.44-1.44$ & & 0.93 & $0.72-1.19$ & \\
\hline$>1.99-2.99$ & 0.44 & $0.18-1.09$ & & 0.76 & $0.56-1.03$ & \\
\hline$>2.99-3.99$ & 0.53 & $0.17-1.59$ & & 0.77 & $0.54-1.09$ & \\
\hline$>4$ & 0.53 & $0.16-1.73$ & & 0.71 & $0.48-1.04$ & \\
\hline \multicolumn{7}{|l|}{ Years per child } \\
\hline 0 & 1.00 & & 0.25 & 1.00 & & 0.44 \\
\hline$>0-0.49$ & 0.97 & $0.52-1.82$ & & 0.94 & $0.71-1.25$ & \\
\hline$>0.49-0.99$ & 0.81 & $0.49-1.33$ & & 0.86 & $0.68-1.08$ & \\
\hline$>1$ & 0.73 & $0.40-1.34$ & & 0.92 & $0.71-1.17$ & \\
\hline
\end{tabular}

Values are adjusted for age, daily energy intake, BMI, WHR, number of live births, smoking, alcohol consumption, physical activity, education, income, occupation and hypertension

cases of type 2 diabetes mellitus. Finally, we found that the beneficial effect associated with the duration of breastfeeding was more evident among women over 60 years of age (data not shown).

\section{Discussion}

In this large, prospective population-based study of middleaged Chinese women, breast-feeding was associated with a reduced risk of type 2 diabetes mellitus [13] independently of known risk factors for type 2 diabetes mellitus.

Our study adds to the limited data on the association between breast-feeding and risk of type 2 diabetes mellitus in mothers. Longer duration of breast-feeding was associated with reduced incidence of type 2 diabetes mellitus in two large US cohorts of young and middle-aged women, the Nurses' Health Studies I and II [6]. Similar to our results, the RRs in these studies for parous women with 0 , $>0$ to 3 months, $>3$ to 6 months, $>6$ to 11 months, $>11$ to 
23 months and $\geq 23$ months of breast-feeding were 1.00 , $0.98,1.03,0.96,0.92$ and 0.88 ( $p=0.02$ for trend $)$ for middle-aged women and 1.00, 1.04, 0.91, 0.87, 0.88 and 0.67 ( $p<0.01$ for trend) for younger women. The RR for women who had breastfed compared with women who had never breastfed was 0.97 (95\% CI, 0.91-1.02) for middleaged women and 0.90 (95\% CI, 0.77-1.04) for younger women. In agreement with our findings, these studies also found that the protection conferred by breast-feeding appeared to wane with time since last birth and that longer duration of breast-feeding per pregnancy was associated with a greater benefit. However, among women with gestational diabetes, breast-feeding was not associated with lower risk of type 2 diabetes mellitus later in life [6]. To our knowledge, no other studies have examined the long-term association between breast-feeding and subsequent development of type 2 diabetes mellitus.

In the Nurses' Health Studies I and II, analyses were adjusted for participants' birthweight and BMI at 18 years, as the investigators found that duration of breast-feeding was inversely related to BMI at 18 years [6]. In our study, we only had information on birthweight for a subset of participants. The correlation coefficients between duration of breast-feeding and both birthweight of participants and BMI at 20 years were 0.02 and 0.12 , respectively. We chose to adjust for current BMI and WHR in our analysis, rather than BMI at 20 years or weight at 20 years, as BMI and WHR were strongly associated with type 2 diabetes mellitus. In addition, measurements used to calculate BMI and WHR in our study were taken by trained professionals at the time of the interview, while weight at 20 years was self-reported. In analyses adjusted for BMI at 20 years, the fully adjusted RRs for $0,>0$ to $0.99,>0.99$ to $1.99,>1.99$ to $2.99,>2.99$ to 3.99 and $\geq 4$ years of breast-feeding were $1.00,0.91,1.02,1.09,0.77$ and 0.73 ( $p=0.30$ for trend). We conducted additional analyses adjusting for standardised weight gain ( $\mathrm{kg}$ per year) since age 20 years and weight at age 20 years. The fully adjusted RRs for $0,>0$ to 0.99 , $>0.99$ to $1.99,>1.99$ to $2.99,>2.99$ to 3.99 and $\geq 4$ years of breast-feeding were $1.00,0.90,0.96,0.99,0.76$ and 0.68 ( $p=0.08$ for trend).

It has been suggested that breast-feeding may protect against type 2 diabetes mellitus by facilitating weight loss, although the association between breast-feeding and weight loss remains inconclusive [14-20]. In our population, the age-adjusted means of standard weight gain since age 20 years by months of breast-feeding were $0.34,0.31,0.33$, 0.36 and $0.37 \mathrm{~kg}$ per year for $0,>0$ to 6 months, $>6$ to 11 months, $>11$ to 35 months and $\geq 36$ months of breastfeeding, respectively $(p<0.01)$. Thus, weight loss is unlikely to be the reason for the inverse association between breast-feeding and type 2 diabetes mellitus observed in our study.
Another possible mechanism is that breast-feeding may improve insulin sensitivity and glucose intolerance. In a study of both breast-feeding and non-breast-feeding nondiabetic women, insulin levels and insulin/glucose ratios were lower, while carbohydrate use and total energy expenditure were higher in the breast-feeding group [21]. Data from studies of women with gestational diabetes suggest that breast-feeding affects insulin and glucose homeostasis. In a study of 809 Latina women, breastfeeding was associated with improved glucose tolerance, fasting glucose and total area under the glucose tolerance curve [2], while in another study of 26 white women (14 breast-feeding, 12 non-breast-feeding), the breast-feeding group had a higher disposition index, indicating more efficient pancreatic beta cell function [3]. Data from animal studies also suggests that in the post-partum period, breastfeeding is associated with a decrease in insulin resistance. Blood glucose levels were reduced $20 \%$ and insulin levels were reduced $35 \%$ in lactating rats compared with nonlactating rats [4], while in another study, a 12-fold increase in insulin uptake was observed in the mammary glands of lactating rats, as well as a marked decrease in the plasma half-life of insulin [5]. Breast-feeding may also influence pituitary hormones [22] and may induce long-term changes in the hypothalamic-pituitary axis [23].

Our study has several strengths. Our population is representative of urban Shanghai. The high follow-up rates minimised the possibility of selection bias. In addition, the prevalence of breast-feeding in this population was very high $(81.62 \%)$, and there was a high number of live births (of the total number of women in our study at baseline, 74,942 or $96.4 \%$ were parous). In addition, the extensive information on potential confounders and the large study size allowed us to examine the effect of duration of breastfeeding on the development of type 2 diabetes mellitus in detail.

The major limitation of the study is reliance on selfreported diabetes, which is an important factor to consider when interpreting these results. A recent report suggested that the prevalence of diabetes was under-diagnosed in Shanghai [13]. We are not aware of any programme for systematic screening for diabetes in our study area. At baseline recruitment, we conducted a urinary glucose test for all cohort members who donated a urine sample $(88.2 \%$ of participants). We found that $1 \%$ of participants who reported never having been diagnosed by a physician as having diabetes had a positive urinary glucose test. These participants were excluded from the current analysis. However, it is possible some other type 2 diabetes cases remained undiagnosed in our study. Similarly, self-reported diabetes may also include some false positive cases. Misclassification of diabetes could weaken the association between duration of breast-feeding and the risk of type 2 
diabetes mellitus. To address the possibility of surveillance bias, we conducted analyses restricted to women with confirmed diabetes and found similar results.

In our study, duration of breast-feeding was correlated with parity. In a recent study from the UK, having more children was associated with a higher risk of diabetes in women [24]. However, the association was attenuated after adjustment for BMI and socioeconomic factors. Similarly to the UK study, we found parity to be associated with lower income and education levels, higher BMI, WHR and standard weight gain, as well as smoking and alcohol consumption in this population. In age-adjusted analyses the incidence of diabetes was positively associated with number of children. The RRs of diabetes were 1.00, 1.38, $1.32,1.31$ and 1.62 for one, two, three, four and five or more children $(p<0.01)$. When we adjusted the analysis for BMI and WHR, parity was no longer associated with incidence of type 2 diabetes. We found that the association between breast-feeding and type 2 diabetes did not change much after adjustment for number of live births. Before and after inclusion of the number of live births in the analysis, the RRs of type 2 diabetes mellitus for $0,>0$ to 6 months, $>6$ to 11 months, $>11$ to 35 months and $\geq 36$ months of breast-feeding were $1.00,0.87,0.89,0.89$ and $0.75(p=0.04$ for trend) and 1.00, $0.87,0.89,0.89$ and $0.73(p=0.05$ for trend), respectively.

Breast-feeding could also reflect differences in other risk factors in our population that might not have been completely controlled for or measured in our study. We applied a propensity score adjustment, including a wide array of lifestyle and dietary intake variables, and found little change in the pattern of associations between breastfeeding and type 2 diabetes mellitus (data not shown). Nevertheless, residual confounding from unmeasured or less than perfectly measured confounders, such as socioeconomic status at the time of breast-feeding, is still a concern. Thus, our results should be interpreted with caution, particularly given that the association was relatively weak and that the reduced risk was limited to participants with a longer duration of breast-feeding ( $\geq 36$ months). In addition, over-adjustment due to the inclusion of possible mediating variables such as BMI and WHR in the model may also be a concern. In our study population, breastfeeding was weakly associated with BMI and WHR ( $r=$ 0.22 and $r=0.22$, respectively). Further studies conducted in other populations and focused on the underlying biological mechanisms are warranted.

In summary, we found that breast-feeding duration was inversely associated with the risk of type 2 diabetes mellitus in this population. Together with results from two other large US cohorts and some clinical evidence of improved glucose homeostasis in breast-feeding women, these data suggest that breast-feeding may reduce the risk of type 2 diabetes mellitus in middle-aged women.

Acknowledgements This study was supported by United States Public Health Service grant number R01 CA070867 from the National Institutes of Health. The authors thank the research staff and participants of the SWHS and B. Hull for technical assistance in the preparation of this manuscript.

Duality of interest The authors declare that there is no duality of interest associated with this manuscript.

\section{References}

1. Zimmet P (2000) Globalization, coca-colonization and the chronic disease epidemic: can the Doomsday scenario be averted? J Intern Med 247:301-310

2. Kjos SL, Henry O, Lee RM, Buchanan TA, Mishell DR Jr (1993) The effect of lactation on glucose and lipid metabolism in women with recent gestational diabetes. Obstet Gynecol 82:451-455

3. McManus RM, Cunningham I, Watson A, Harker L, Finegood DT (2001) Beta-cell function and visceral fat in lactating women with a history of gestational diabetes. Metabolism 50:715-719

4. Burnol AF, Leturque A, Ferre P, Kande J, Girard J (1986) Increased insulin sensitivity and responsiveness during lactation in rats. Am J Physiol 251:E537-E541

5. Jones RG, Ilic V, Williamson DH (1984) Physiological significance of altered insulin metabolism in the conscious rat during lactation. Biochem J 220:455-460

6. Stuebe AM, Rich-Edwards JW, Willett WC, Manson JE, Michels KB (2005) Duration of lactation and incidence of type 2 diabetes. JAMA 294:2601-2610

7. Zheng W, Chow WH, Yang G et al (2005) The Shanghai Women's Health Study: rationale, study design, and baseline characteristics. Am J Epidemiol 162:1123-1131

8. Zhang X, Shu XO, Gao YT et al (2004) Anthropometric predictors of coronary heart disease in Chinese women. Int $\mathrm{J}$ Obes Relat Metab Disord 28:734-740

9. Matthews CE, Shu XO, Yang G et al (2003) Reproducibility and validity of the Shanghai Women's Health Study physical activity questionnaire. Am J Epidemiol 158:1114-1122

10. Ainsworth BE, Haskell WL, Whitt MC et al (2000) Compendium of physical activities: an update of activity codes and MET intensities. Med Sci Sports Exerc 32(Suppl):S498-S504

11. Shu XO, Yang G, Jin F et al (2004) Validity and reproducibility of the food frequency questionnaire used in the Shanghai Women's Health Study. Eur J Clin Nutr 58:17-23

12. Yang YX, Wang GY, Pan XC (2002) Chinese food composition tables. Peking University Medical Press, Beijing

13. Jia WP, Pang C, Chen L et al (2007) Epidemiological characteristics of diabetes mellitus and impaired glucose regulation in a Chinese adult population: the Shanghai Diabetes Studies, a crosssectional 3-year follow-up study in Shanghai urban communities. Diabetologia 50:286-292

14. Brewer MM, Bates MR, Vannoy LP (1989) Postpartum changes in maternal weight and body fat depots in lactating vs nonlactating women. Am J Clin Nutr 49:259-265

15. Dugdale AE, Eaton-Evans J (1989) The effect of lactation and other factors on post-partum changes in body-weight and triceps skinfold thickness. Br J Nutr 61:149-153 
16. Butte NF, Hopkinson JM (1998) Body composition changes during lactation are highly variable among women. J Nutr $128(2$ Suppl):381S-385S

17. Sichieri R, Field AE, Rich-Edwards J, Willett WC (2003) Prospective assessment of exclusive breastfeeding in relation to weight change in women. Int J Obes Relat Metab Disord 27:815-820

18. Valeggia CR, Ellison PT (2003) Impact of breastfeeding on anthropometric changes in peri-urban Toba women (Argentina). Am J Hum Biol 15:717-724

19. Dewey KG, Heinig MJ, Nommsen LA (1993) Maternal weight-loss patterns during prolonged lactation. Am J Clin Nutr 58:162-166

20. Gigante DP, Victora CG, Barros FC (2001) Breast-feeding has a limited long-term effect on anthropometry and body composition of Brazilian mothers. J Nutr 131:78-84
21. Butte NF, Wong WW, Hopkinson JM (2001) Energy requirements of lactating women derived from doubly labeled water and milk energy output. J Nutr 131:53-58

22. de Zegher F, Spitz B, Van den Berghe G et al (1998) Postpartum hyperprolactinemia and hyporesponsiveness of growth hormone (GH) to GH-releasing peptide. J Clin Endocrinol Metab 83:103106

23. Lankarani-Fard A, Kritz-Silverstein D, Barrett-Connor E, GoodmanGruen D (2001) Cumulative duration of breast-feeding influences cortisol levels in postmenopausal women. J Womens Health Gend Based Med 10:681-687

24. Hardy R, Lawlor DA, Black S, Wadsworth ME, Kuh D (2007) Number of children and coronary heart disease risk factors in men and women from a British birth cohort. BJOG 114:721-730 